\title{
Current-limiting characteristics of saturated iron-core fault current limiters in VSC-HVDC systems based on electromagnetic energy conversion mechanism
}

\author{
Botong $\mathrm{LI}^{1}$, Hanqing CUI ${ }^{1}$, Fangjie $\mathrm{JING}^{1}$, Bin $\mathrm{LI}^{1}$, Yichao $\mathrm{LIU}^{1}$
}

MPCE

\begin{abstract}
A common method to examine the current-limiting performance of saturated iron-core fault current limiter (SI-FCL) in high-voltage direct-current transmission based on voltage source converter (VSC-HVDC) systems is to solve differential equations based on the system fault transient characteristics and the equivalent inductance calculation equation. This method analyzes the fault current of the VSC-HVDC system in the time domain. However, it is computationally complex and cannot directly reflect the relationship between parameters and the currentlimiting effect of the SI-FCL. In this paper, the relationship between the magnetic flux density and magnetic field energy of the SI-FCL is analyzed. The energy exchange between the DC capacitor and the SI-FCL in the DC short circuit fault process is analyzed. From the perspective of electromagnetic energy conversion, the criterion for determining the current-limiting ability of the SI-FCL in the transient process is given based on the parameters of
\end{abstract}

CrossCheck date: 13 July 2018

Received: 10 November 2017/Accepted: 13 July 2018/Published online: 16 October 2018

(C) The Author(s) 2018

$\triangle$ Botong LI

libotong@tju.edu.cn

Hanqing CUI

cuihanqing12@163.com

Fangjie JING

jing_fangjie@126.com

Bin LI

binli@tju.edu.cn

Yichao LIU

liuyichao@tju.edu.cn

1 Key Laboratory of Smart Grid of Ministry of Education, Tianjin University, Tianjin 300072, China the SI-FCL and VSC-HVDC system. On this basis, the characteristics of the DC side fault current and the capacitor voltage when the SI-FCL has current-limiting ability are examined. Based on the parameters of the SI-FCL and VSC-HVDC system, a method for calculating the fault current peak value and capacitor voltage drop time is given. Finally, the accuracy of the analysis of the SI-FCL in the VSC-HVDC system based on the electromagnetic energy conversion mechanism is demonstrated through a case study and simulation results of the VSC-HVDC system with different SI-FCLs.

Keywords Electromagnetic energy, Saturated iron-core fault current limiter (SI-FCL), High-voltage direct-current transmission based on voltage source converter (VSC-

HVDC) system, Fault analysis

\section{Introduction}

High-voltage direct-current transmission based on voltage source converter (VSC-HVDC) systems are a new type of DC transmission technology with fully controllable power electronic devices, which is one key technology in the research and development of the smart grid [1]. The characteristics of the converter structure and the control strategy of the VSC-HVDC system result in high peak value and fast rising speed of the DC side fault current. A large fault current can easily damage converter components and protective devices [2,3], yet is difficult to eliminate immediately. Therefore, current-limiting technology is introduced to suppress the DC side fault current, and slow down the capacitor voltage drops. Meanwhile, this technology can effectively protect equipment [4], relax the requirements on the DC breaker in terms of the speed and 
capacity, and reduce the difficulty of designing the protection scheme.

As current-limiting technology is critical to the VSCHVDC system, much related research has been performed and results achieved. Reference [5] analyzed the influence of the location of resistance-type superconductive fault current limiters (SFCLs) on the DC side fault. They pointed out that installing the resistance-type SFCL on the DC side could successfully limit the DC fault current in the case of a DC line fault. Reference [6] studied the current-limiting effect of the resistance-type SFCL in the single-pole ground fault and inter-pole fault at the DC side, and the simulation results revealed that the resistance-type SFCL has a significant effect on the DC side fault. Reference [7] examined application cases of the resistance-type SFCL in a three-terminal VSC-HVDC system, and proved the limiting effect of this STCL on various types of DC side fault current. Reference [8] analyzed the performance of the resistance-type SFCL in a multi-terminal VSC-HVDC system connected with an offshore wind farm, and their transient analysis for different perturbations proved that the resistance-type SFCL can reduce the fault current for both DC and AC faults. Reference [9] analyzed the coordination of fault current limitation in an electric power grid with multiple SFCLs to further limit fault current rise. Reference [10] proposed a new resistance-type SFCL that can reduce the fault current to $50 \%$ of the original value within $2 \mathrm{~ms}$. To limit the fault current, the resistance of the fault circuit is increased by installing a resistance-type SFCL in the VSC-HVDC system.

Some literatures have also proposed limiting the fault current by increasing the circuit inductance [11]. Reference [12] designed an inductive SFCL and the simulation results revealed that this SFCL has a significant effect on the DC side fault. Reference [13] analyzed the impact of different inductance values in the VSC-HVDC system on the DC side fault current. Reference [14] offered an optimal design scheme of installing the current-limiting inductor in a multi-terminal VSC-HVDC system, and proved that this scheme can effectively deaccelerate the rise in fault current. Reference [15] designed a protective inductor for the VSC-HVDC system of an offshore wind farm to limit the current. The coordination relationships among converter, DC circuit breaker and DC SFCL were studied and some technical indicators were proposed to evaluate the performance of the SFCL in [16].

Though inductors at the DC side can serve as current limiters, large reactance will reduce the dynamic response speed and can easily cause parallel resonance. In normal operation, the equivalent inductance of saturated iron-core fault current limiters (SI-FCL) is so small that it has no effect on the VSC-HVDC system. When a DC side fault occurs, the equivalent inductance increases rapidly to limit the fault current. Thus, this approach is superior to installing inductors.

Some researches have also investigated the currentlimiting effect of the SI-FCL in the VSC-HVDC system. Reference [11] proved the current-limiting effect of the SIFCL in the VSC-HVDC system. Reference [17] analyzed the transient process of the DC side fault in the VSCHVDC system with the SI-FCL, and gave the relationship between the peak value of the fault current and the SI-FCL parameters. Reference [18] analyzed the leakage magnetic flux of the SI-SFCL and proposed the equivalent magnetic circuit model considering the effect of leakage magnetic flux. Reference [19] introduced the application of the active SI-SFCL in the VSC-HVDC system.

The analysis method in these papers solves the DC side circuit differential equation with the equivalent inductance of the SI-FCL in the DC side fault condition. However, owing to the nonlinearity of the equivalent inductance, the calculation involved is enormous and error prone. Other researches mainly focused on the application of the SI-FCL to the AC system, which is not applicable to the VSCHVDC system [20-22].

To analyze the current-limiting effect of the SI-FCL in the VSC-HVDC system, this paper analyzes the energy exchange between the SI-FCL and the DC capacitor after the DC side fault occurs. The relationship between the DC side fault current and the parameters of the SI-FCL is studied, and a rapid and effective analytical method of the current-limiting performance of the SI-FCL is proposed in this paper.

\section{Analysis of energy variation process of SI-FCL with changing magnetic flux density}

The basic structure of the SI-FCL is shown in Fig. 1, which includes one iron-core, one coil connected with the DC side of the VSC-HVDC system, and one excitation coil with a DC-biased source circuit. To reduce the run-time

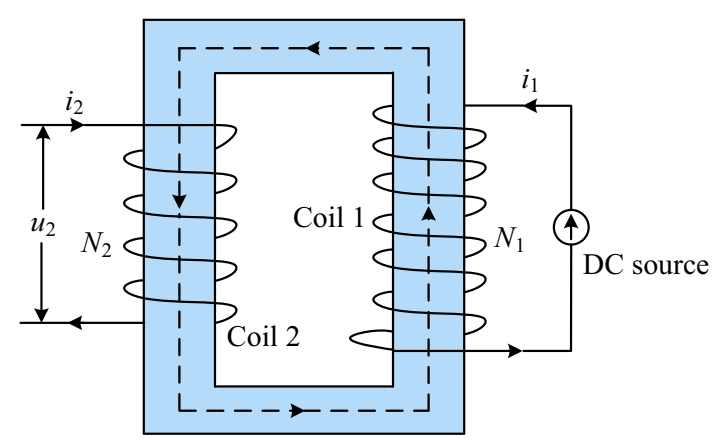

Fig. 1 Structure of SI-FCL for VSC-HVDC system 
loss, the excitation coil can be made of superconductive materials.

In Fig. 1, coil 1 is the excitation coil and coil 2 is the coil connected with the DC side of the VSC-HVDC system; $N_{1}$ is the turn number of coil 1 and $N_{2}$ is the turn number of coil $2 ; i_{1}$ is the current of coil 1 generally excited by a constant current source; thus, $i_{1}$ is considered as constant; $i_{2}$ is the current of the DC side of the VSCHVDC system.

The topology of the VSC-HVDC system with SI-FCLs is shown in Fig. 2. To limit the DC side fault current, SIFCLs are installed in the converter station export. The locations are also shown in Fig. 2.

As shown in Fig. 3, the magnetic flux density-magnetic field intensity $B-H$ curve of the iron core used in the SISFCL is divided into the saturated region and the unsaturated region signified by points $K_{1}$ and $K_{2}$. When the VSCHVDC system works normally, the magnetic flux density of the iron core is in the positive saturated region, the permeability of the iron core is small, and the equivalent inductance of the SI-FCL is extremely small; thus, its influence on the VSC-HVDC system is slight. When a DC side short-circuit fault occurs, the greatly increased fault current forces the magnetic flux density of the core into the non-saturated region. Subsequently, the equivalent inductance of the SI-FCL rises rapidly with the increase in permeability, and the fault current is limited by the SIFCL.

From the perspective of electromagnetic energy conservation, the electric energy is absorbed and converted into magnetic field energy by the SI-FCL after a DC side fault. Thereafter, the magnetic field energy draws the magnetic flux density from the positive saturated region into the unsaturated region. During this process, the magnetic permeability of the iron core increases, and so does the equivalent inductance of the SI-FCL. The energy conversion of the SI-FCL is analyzed at the stage when the magnetic flux density moves from the positive saturated region into the unsaturated region.

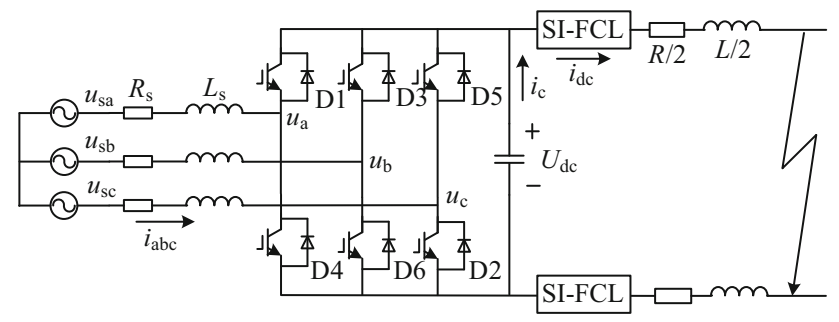

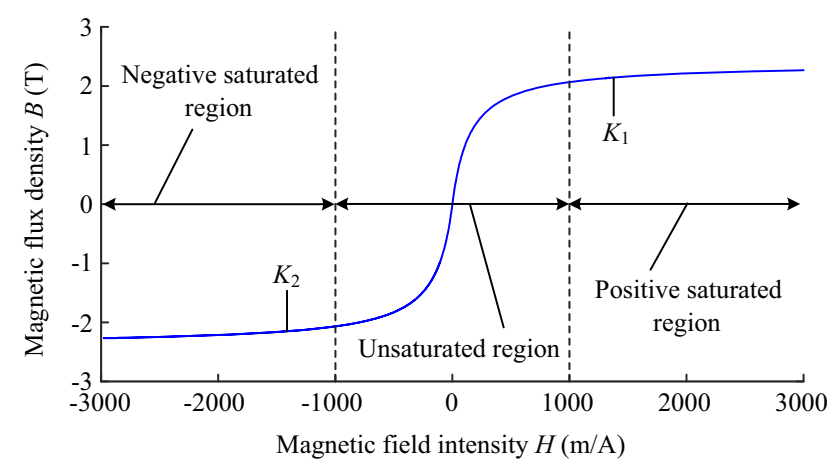

Fig. $3 B-H$ curve of iron core

\subsection{Theoretical analysis of energy change of SI-FCL with varying magnetic flux density}

For simplicity, it is assumed that the cross-section areas of the iron core denoted by $S$ are identical. The magnetic flux density is $B$. The relationship between the magnetic flux density $B$ and the current of the DC side of the VSCHVDC system $i_{2}$ can be expressed by the following equation according to the relevant knowledge of magnetic circuit analysis [23]:

$i_{2}=\frac{N_{1} i_{1}-H l}{N_{2}}$

where $l$ is the overall length of the core; $H$ is the magnetic field intensity. From the energy calculation formula, we can see that the energy variation of the SI-FCL in the period of $t_{1}-t_{2}$ is:

$\Delta W_{L}=\int_{t_{1}}^{t_{2}} u_{2} i_{2} \mathrm{~d} t=S l \int_{B_{1}}^{B_{2}} \frac{B}{\mu} \mathrm{d} B-S N_{1} i_{1} B_{2}+S N_{1} i_{1} B_{1}$

where $B_{1}$ is the magnetic flux density at time $t_{1} ; B_{2}$ is the magnetic flux density at time $t_{2} ; S$ is the cross-section of the iron core.

According to the $B-H$ curve in Fig. 3, the energy variation of the SI-FCL after the fault is:

$$
\begin{aligned}
\Delta W_{L}= & S l \int_{B_{m}}^{B_{k}} \frac{B}{\mu} \mathrm{d} B-S N_{1} i_{1} B_{k}+S N_{1} i_{1} B_{m} \\
& +S l \int_{B_{k}}^{B_{t}} \frac{B}{\mu} \mathrm{d} B-S N_{1} i_{1} B_{t}+S N_{1} i_{1} B_{k}
\end{aligned}
$$

where $B_{m}$ is the magnetic flux density before the fault and in the saturated region; $B_{t}$ is the magnetic flux density at a time point after the fault and in the non-saturated region; $B_{k}$ is the magnetic flux density of point $K_{1}$.

Because the variation of the magnetic flux density in the saturated region is negligible, $B_{m} \approx B_{k}$. Hence, (3) can be simplified as follow:

Fig. 2 Topology of VSC-HVDC system with SI-FCLs 
$\Delta W_{L}=S l \int_{B_{k}}^{B_{t}} \frac{B}{\mu} \mathrm{d} B-S N_{1} i_{1} B_{t}+S N_{1} i_{1} B_{k}$

According to the $B-H$ curve of the iron core in Fig. 3, we obtain the permeability-magnetic flux density $\mu_{1}-$ $B$ curve shown in Fig. 4 that depicts the $\mu-B$ relationship of the core. It can be seen from the Fig. 4 , that the $\mu-B$ curve is linear in the unsaturated region.

Therefore, the first-order polynomial can be used to fit the relationship between the permeability $\mu$ and the magnetic flux density $B$ with high accuracy. The fitting equation is:

$\mu= \begin{cases}m B+n & 0 \leq B \leq B_{k} \\ -m B+n & -B_{k} \leq B<0\end{cases}$

where the values of $m$ and $n$ depend on the material of the iron core.

The $\mu_{2}-B$ curve in Fig. 4 is obtained from (5). Compared with the $\mu_{1}-B$ curve, it can be seen that (5) can well fit the non-saturated region of the $\mu-B$ curve, but is not consistent with the saturated region.

According to (3) and (5), when the magnetic flux density $B$ changes from the positive saturation region to the unsaturated region, the relationship between the energy variation $\Delta W_{L}$ and the magnetic flux density $B_{t}$ is:

$$
\Delta W_{L}=\left\{\begin{array}{lc}
\frac{S \operatorname{lm}}{k^{2}} \ln \left(\frac{\mu_{B_{k}}}{k \mu_{B_{t}}+m}\right)-S \frac{l}{k}\left(B_{k}-B_{t}\right) \\
+S N_{1} i_{1}\left(B_{k}-B_{t}\right) & 0 \leq B_{t} \leq B_{k} \\
\frac{S \ln }{k^{2}} \ln \left(\frac{\mu_{B_{k}}}{-k \mu_{B_{t}}+m}\right) & -S \frac{l}{k}\left(B_{k}+B_{t}\right) \\
+S N_{1} i_{1}\left(B_{k}-B_{t}\right) & -B_{k} \leq B_{t}<0
\end{array}\right.
$$

where $\mu_{B k}$ is the permeability when the magnetic flux density is $B_{k}$.

When the magnetic flux density is in the unsaturated region, the relationship between the energy variation and the magnetic flux density can be reflected by (6). Thus, the variation of magnetic field energy can be calculated by (6)

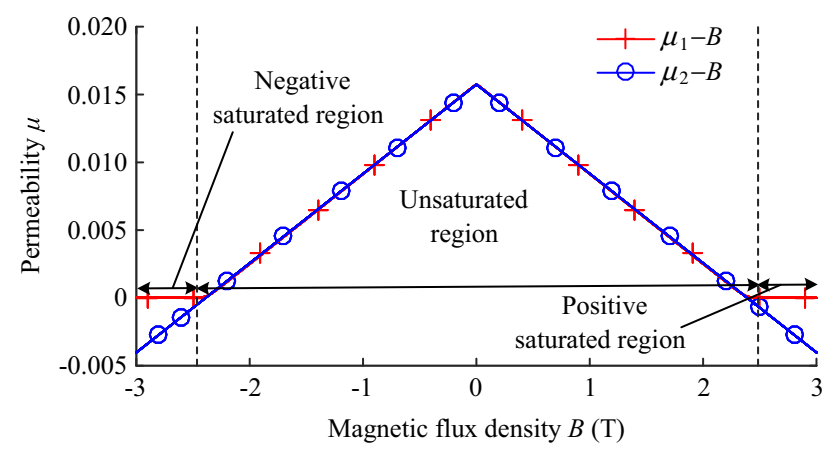

only when the magnetic flux density varies from the knee point to the unsaturated region.

\subsection{Simulation and verification of SI-FCL energy change with varying magnetic flux density}

To verify the correctness of (6), a simulation model of the SI-FCL is established in PSpice. A single-phase transformer model with saturated characteristics was built to simulate the SI-FCL, and the parameters of the model are listed in Table 1.

The excitation coil is connected to a ramp-type current source that generates current to drive the magnetic flux density of the SI-FCL from the positive saturated region across the unsaturated region into the negative saturated region. Current $i_{2}$, voltage $u_{2}$, and the magnetic flux density are recorded constantly in the simulation. According to (2), the energy variation of the SI-FCL from the simulation start time to a certain time can be calculated, and then the relationship between the energy variation and magnetic flux density can be obtained, which is shown as the $\Delta W_{L 1^{-}}$ $B$ curve in Fig. 5 with cross marks.

Based on (6), the relationship between the energy variation and the magnetic flux density is calculated and shown as the $\Delta W_{L 2}-B$ curve in Fig. 5 with circle marks.

In Fig. 5, the two curves coincide well when the magnetic flux density varies from the positive saturated region near the point $K_{1}$ to the unsaturated region. Equation (6) proves to be accurate in calculating the energy variation of the SI-FCL with the magnetic flux density ranging from the positive saturated region to the unsaturated region.

\section{Current-limiting performance analysis of SI- FCL in VSC-HVDC system}

The structure of the two-level VSC-HVDC system is shown in Fig. 2. The DC side faults in the system can be categorized as two-pole short-circuit fault, single-pole short-circuit fault and disconnection fault, among which

Table 1 Parameters of SI-FCL simulation model

\begin{tabular}{ll}
\hline Parameter & Value \\
\hline Length of the core $(l)$ & $6 \mathrm{~m}$ \\
Cross-section areas $(S)$ & $1.1 \mathrm{~m}^{2}$ \\
Winding number of coil 1 $\left(N_{1}\right)$ & 1500 \\
Winding number of coil 2 $\left(N_{2}\right)$ & 150 \\
Current of the excitation coil $\left(i_{1}\right)$ & $30 \mathrm{~A}$ \\
Magnetic flux density of point $K\left(B_{k}\right)$ & $2.3 \mathrm{~T}$ \\
\hline
\end{tabular}

Fig. $4 \mu-B$ curve of iron core 


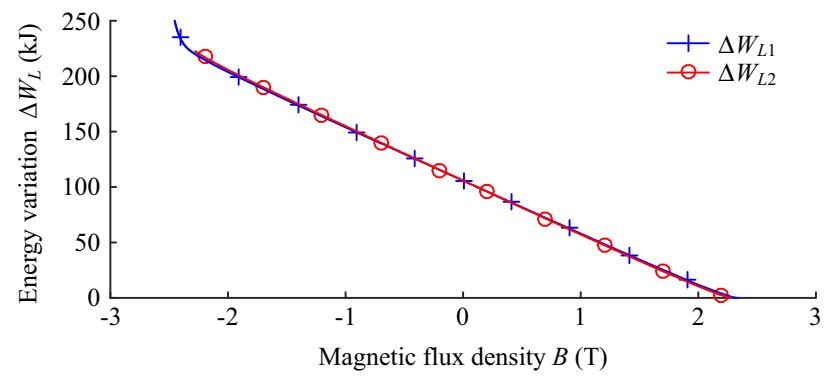

Fig. 5 Relationship between energy variation and magnetic flux density

the two-pole short-circuit fault is the most harmful. Therefore, this paper analyzes the energy exchange process of the DC side in the case of a two-pole short-circuit fault. On this basis, the current-limiting performance of the SIFCL is studied.

When a DC side fault occurs, the insulated-gate bipolar transistors of the inverter will be blocked under self-protection. At this time, the capacitor voltage $U_{\mathrm{dc}}$ is greater than the peak value of the inter-phase voltage of the AC side. Additionally, the AC side system cannot supply power to the DC side system through the free-wheel diodes. The DC side system can be regarded as a circuit structure in which the capacitor discharges to the shortcircuit point. After the capacitor voltage drops below the peak value of the inter-phase voltage of the AC side, the free-wheel diodes will turn on, and will most likely be damaged by the fault current [3]. Therefore, the SI-FCL that provides ample time for the breaker to clear the fault should limit the fault current and delay the voltage drop time of the capacitor before the free-wheel diodes turns on.

In the most serious scenario, in which the resistance of the SI-FCL ignored, and a short-circuit fault occurs at the junction of the DC rail and the power cable, the DC side system is equivalent to the simplified circuit as shown in Fig. 6 until the free-wheel diodes turn on.

For the circuit shown in Fig. 6, the capacitor voltage at initial time $t_{1}$ is denoted as $U_{\mathrm{dc} 1}$, a time point after the fault is denoted as $t_{2}$, and the voltage at time $t_{2}$ is denoted as $U_{\mathrm{dc} 2}$. The energy variation of the capacitor in the period $t_{1}-$ $t_{2}$ is:

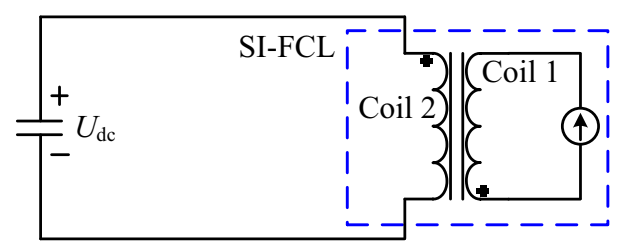

Fig. 6 Equivalent circuit of DC side system after a two-pole shortcircuit fault
$\Delta W_{c}=\frac{1}{2} C U_{\mathrm{dc} 1}^{2}-\frac{1}{2} C U_{\mathrm{dc} 2}^{2}$

As observed from the equivalent circuit in Fig. 6, the electric field energy variation of the capacitor is equal to the magnetic field energy variation of the SI-FCL, $\Delta W_{c}=$ $\Delta W_{L}$. According to the relationship between $\Delta W_{L}$ and the magnetic flux density $B$ shown in Fig. 5 , it can be seen that the magnetic flux density $B$ will gradually alter from the positive saturated region to the negative saturated region as the energy variation increases.

As can be seen from the $B-H$ curve shown in Fig. 3, the magnetic field intensity $H$ is reduced rapidly when the magnetic flux density $B$ changes from the positive saturated region to the unsaturated region. Meanwhile, the current $i_{2}$ increases rapidly, which can be seen from (1). When the magnetic flux density $B$ is in the unsaturated region, the magnetic field intensity $H$ converts slowly, and the current $i_{2}$ is basically stable at a fixed value. When the magnetic flux density $B$ enters the negative saturated region, the magnetic field intensity $H$ will decrease rapidly again, the current $i_{2}$ will dramatically increase, and the SI-FCL will no longer limit the fault current of the DC side. Therefore, to achieve the desired current-limiting effect, the energy released by the capacitor should remain below what can drive the magnetic flux density $B$ from the positive saturated region across the unsaturated region into the negative saturated region.

Combining (6) and (7), when the magnetic flux density $B$ varies from the positive saturated region into the unsaturated region, the relationship between the energy variation of the SI-FCL and the energy variation of the capacitor is:

$$
\begin{aligned}
& \frac{1}{2} C U_{\mathrm{dc} 1}^{2}-\frac{1}{2} C U_{\mathrm{dc} 2}^{2} \\
& =\left\{\begin{array}{c}
\frac{S \operatorname{lm}}{k^{2}} \ln \left(\frac{\mu_{B_{k}}}{k \mu_{B_{t}}+m}\right)-S \frac{l}{k}\left(B_{k}-B_{t}\right) \\
+S N_{1} i_{1}\left(B_{k}-B_{t}\right) \quad 0 \leq B_{t} \leq B_{k} \\
\frac{S \operatorname{lm}}{k^{2}} \ln \left(\frac{\mu_{B_{k}}}{-k \mu_{B_{t}}+m}\right)-S \frac{l}{k}\left(B_{k}+B_{t}\right) \\
+S N_{1} i_{1}\left(B_{k}-B_{t}\right) \quad-B_{k} \leq B_{t}<0
\end{array}\right.
\end{aligned}
$$

It is noted that in (8) the magnetic flux density $B_{t}$ is in the unsaturated region. Once the magnetic flux density moves from the non-saturated region to the negative saturated region, the $\mu-B$ curve is no longer consistent with (5), and (8) fails to reflect the relationship between the energy variation of the SI-FCL and that of the capacitor.

The capacitor voltage that turns on the free-wheel diodes is $U_{\mathrm{dc}, \mathrm{min}}$. In the extreme case that the capacitor voltage drops to $U_{\mathrm{dc}, \mathrm{min}}$, the energy absorbed by the SI-FCL is not 
sufficient to draw the magnetic flux density $B$ into the negative saturated region, signified by point $K_{2}$.

According to (2), for $B$ not to reach the negative saturated region, the condition is:

$2 S N_{1} i_{1} B_{k} \geq \frac{1}{2} C U_{\mathrm{dc} 1}^{2}-\frac{1}{2} C U_{\mathrm{dc}, \text { min }}^{2}$

Let $\Delta W_{L, \max }=2 S N_{1} i_{1} B_{k}$, then $\Delta W_{L, \max }$ denotes the maximum energy absorbed by the SI-FCL when the magnetic flux density $B$ stays out of the negative saturated region. Let $\Delta W_{c, \max }=1 / 2 C U_{\mathrm{dc} 1}^{2}-1 / 2 C U_{\mathrm{dc}, \text { min }}^{2}$, then $\Delta W_{c, \max }$ represents the energy variation of the capacitor when the voltage drops to $U_{\mathrm{dc}, \min }$, and (9) can be expressed as:

$\Delta W_{L, \max } \geq \Delta W_{c, \max }$

Equation (10) is a criterion used to determine whether the SI-FCL has current-limiting ability all the time before the capacitor voltage drops to $U_{\mathrm{dc} \text {,min }}$.

When the capacitance and voltage level of the VSCHVDC system are given, if the criterion is met, the SI-FCL will have current-limiting ability all the time before the capacitor voltage drops to $U_{\mathrm{dc}, \min }$ and the fault current $i_{2}$ will not surge. Otherwise, the SI-FCL will not always have current-limiting ability and thus cannot achieve the desired performance.

The SI-FCL is designed to limit the fault current to an acceptable level. In addition, the SI-FCL applied in the VSC-HVDC system is also used to extend the time before the voltage drops to $U_{\mathrm{dc}, \min }$. Therefore, in addition to analyzing whether the SI-FCL has current-limiting ability in the process when the capacitor voltage drops from the initial state $U_{\mathrm{dc} 1}$ to $U_{\mathrm{dc} \text {,min }}$, the maximum fault current $\left(i_{2, \max }\right)$ and the duration of this process $(T)$ also needs to be obtained.

When the magnetic flux density $B$ is in the unsaturated region, the current $i_{2}$ can be calculated by (11) derived from (1) and (5).

$i_{2}= \begin{cases}\frac{N_{1} i_{1}-\frac{B l}{k B+m}}{N_{2}} & 0 \leq B \leq B_{k} \\ \frac{N_{1} i_{1}-\frac{B l}{-k B+m}}{N_{2}} & -B_{k} \leq B<0\end{cases}$

Depending on whether (10) is met before the capacitor voltage drops to $U_{\mathrm{dc}, \min }$, the calculation of $i_{2, \max }$ can be divided into two cases:

Case 1: the SI-FCL has current-limiting ability constantly during this voltage drop process. In this case, (8) is used to calculate the magnetic flux density $B_{\min }$ when the capacitor voltage is $U_{\mathrm{dc}, \min }$. With $B_{\min }$ substituted into (11), the maximum fault current $i_{2, \text { max,I }}$ is known.
Case 2: the SI-FCL does not always have current-limiting ability during this process. In this case, the magnetic flux density of the SI-FCL will enter the negative saturated region, after which the fault current will surge before the capacitor voltage drops to $U_{\mathrm{dc}, \min }$. By substituting the magnetic flux density at point $K_{2}$ into (11), the maximum fault current $i_{2, \text { max,II }}$ can be obtained.

In normal operation, the iron core of the SI-FCL works in the saturated state so that the equivalent inductance is small. To ensure that the core is saturated, $N_{1} i_{1}$ must be very large. When a DC side fault occurs, the magnetic flux density enters the unsaturated region. As shown in Fig. 3, when the core is in the unsaturated state, the magnetic field intensity is small and changes little. Meanwhile, the variation range of the current $i_{2}$ is small, and it can be assumed that $i_{2}$ is approximately constant.

According to the capacitor discharge formula, it is known that:

$i_{2}=-C \frac{\mathrm{d} U_{\mathrm{dc}}}{\mathrm{d} t}$

Setting $T$ is the time for the capacitor voltage to fall to $U_{\mathrm{dc} \text {,min }}$. Depending on whether the condition (10) can be satisfied during the process before the capacitor voltage drops to $U_{\mathrm{dc}, \mathrm{min}}$, the calculation of $T$ is divided into two cases:

Case 1: the SI-FCL has current-limiting ability all the time during this process. In this case, the maximum fault current is $i_{2, \max , \mathrm{I}}$. If the fault current is set to $i_{2, \max , \mathrm{I}}$, the shortest time $T_{\mathrm{min}, \mathrm{I}}$ for capacitor voltage to fall from $U_{\mathrm{dc} 1}$ to $U_{\mathrm{dc}, \min }$ is:

$T_{\min , \mathrm{I}}=\frac{C\left(U_{\mathrm{dc} 1}-U_{\mathrm{dc}, \min }\right)}{i_{2, \max , \mathrm{I}}}$

Case 2: the SI-FCL does not always have currentlimiting ability during this process. In this case, by substituting the magnetic flux density at point into (8), the voltage $U_{\mathrm{dc}, \mathrm{s}}$ when the fault current begins to surge can be obtained as:

$U_{\mathrm{dcs}}=\sqrt{U_{\mathrm{dc} 1}^{2}-4 S N_{1} i_{1} B_{k} / C}$

As the variation range of the current $i_{2}$ is narrow, it is

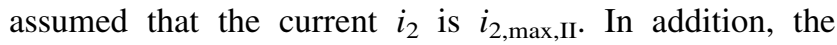
shortest time for capacitor voltage to fall from $U_{\mathrm{dc} 1}$ to $U_{\mathrm{dc}, \mathrm{s}}$ is:

$T_{\min , \mathrm{s}}=\frac{C\left(U_{\mathrm{dc} 1}-U_{\mathrm{dc}, \mathrm{s}}\right)}{i_{2, \max , \mathrm{II}}}$

Assuming that the fault current remains $i_{2, \max , I}$, the shortest time for capacitor voltage to fall from $U_{\mathrm{dc} 1}$ to $U_{\mathrm{dc}, \min }$ is: 
$T_{\text {min }, \mathrm{s}}^{\prime}=\frac{C\left(U_{\mathrm{dc} 1}-U_{\mathrm{dc}, \min }\right)}{i_{2, \max , \mathrm{II}}}$

The time for capacitor voltage to fall from $U_{\mathrm{dc} 1}$ to $U_{\mathrm{dc}, \min }$ is $T_{\text {min,II }}$, and $T_{\text {min,II }}$ is greater than $T_{\text {min,s }}$. After the capacitor voltage drops to $U_{\mathrm{dc}, \mathrm{s}}$, the DC side fault current surges, and $T_{\min , \mathrm{II}}$ must be less than $T_{\min , \mathrm{s}}^{\prime}$. Thus, $T_{\text {min,II }}$ satisfies the condition as follow:

$T_{\min , \mathrm{s}}<T_{\min , \mathrm{II}}<T_{\min , \mathrm{s}}^{\prime}$

Through the analysis of the SI-FCL in the VSC-HVDC system, the criterion is found for determining whether the SI-FCL has current-limiting ability all the time before the capacitor voltage drops to $U_{\mathrm{dc} \text {,min. }}$ By further analyzing the process in which the capacitor voltage drops from the initial state $U_{\mathrm{dc} 1}$ to $U_{\mathrm{dc}, \min }$, the current level to which the fault current is limited, and the time for the capacitor voltage to drop from $U_{\mathrm{dc} 1}$ to $U_{\mathrm{dc}, \min }$ are obtained.

\section{Case study and simulation verification}

To verify the correctness of the theoretical analysis above, a two-level VSC-HVDC system model of $\pm 35 \mathrm{kV}$ $\left(U_{\mathrm{dc}}=70 \mathrm{kV}\right)$ is built in PSpice. The model structure is shown in Fig. 2, and the parameters of the model are listed in Table 2.

The transmission capacity is $20 \mathrm{MW}$, and the rated current $i_{2 \text { rated }}$ is calculated as $0.3 \mathrm{kA}$. For the two-level VSC-HVDC system, the free-wheel diodes turn on once the capacitor voltage drops to the peak value of the interphase voltage at the $\mathrm{AC}$ side, so $U_{\mathrm{dc}, \min }$ is calculated as $49.497 \mathrm{kV}$.

The capacitor voltage and fault current are shown in Figs. 7 and 8, when a two-pole short-circuit fault occurs 5 $\mathrm{km}$ away from the rectifier at $0.1 \mathrm{~s}$. It can be seen that the capacitor voltage drops rapidly to zero without the SI-FCL

Table 2 Parameters of the two-level VSC-HVDC system model

\begin{tabular}{ll}
\hline Parameter & Value \\
\hline DC side voltage & $\pm 35 \mathrm{kV}$ \\
RMS inter-phase voltage of the AC side & $35 \mathrm{kV}$ \\
Length of the DC side cable & $10 \mathrm{~km}$ \\
Transmission capacity & $20 \mathrm{MW}$ \\
Resistance of the DC cable & $0.15 \Omega / \mathrm{km}$ \\
Inductance of the DC cable & $2.5 \mathrm{mH} / \mathrm{km}$ \\
Inductance of the AC side & $0.01 \mathrm{H}$ \\
DC capacitor & $2.0 \mathrm{mF}$ \\
\hline
\end{tabular}

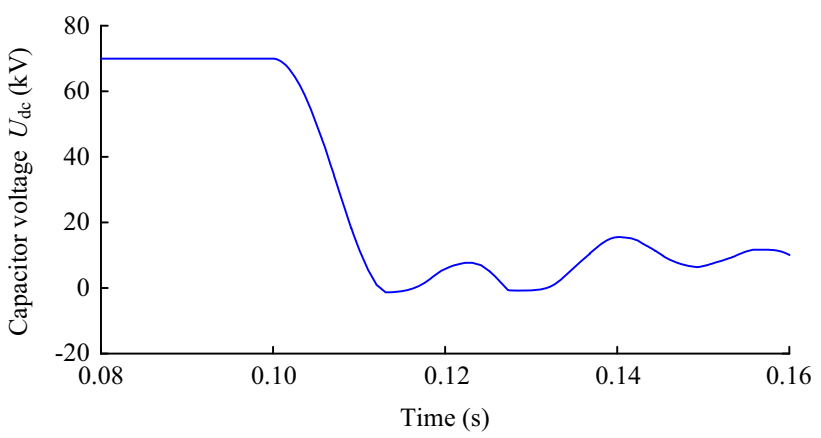

Fig. 7 Capacitor voltage of the VSC-HVDC system without an SIFCL

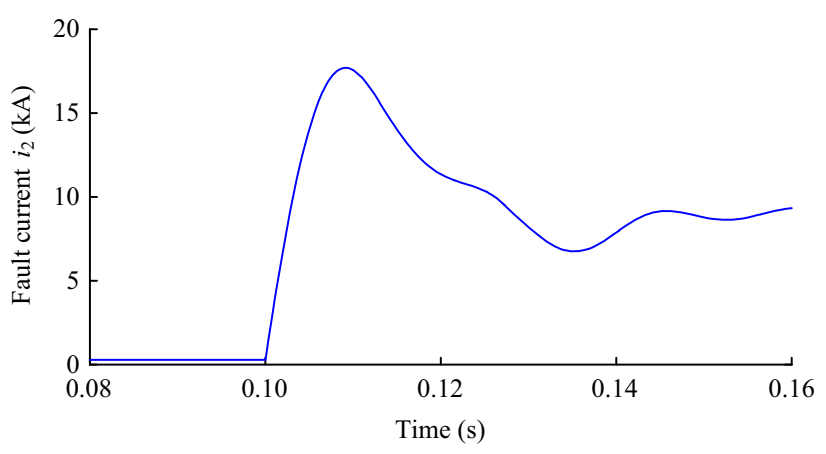

Fig. 8 DC side fault current of VSC-HVDC system without an SIFCL

while the fault current rises rapidly and reaches $17.69 \mathrm{kA}$, approximately 60 times higher than the rated current.

To verify the correctness of the theoretical analysis of the SI-FCL, the SI-FCLs A and B are separately connected in the VSC-HVDC system. The parameters of SI-FCL A are listed in Table 3.

The current-limiting performance of SI-FCL A and B can be analyzed by the theory presented in Section 3 .

Assuming that the two-pole short-circuit fault occurs at the junction of the DC rail and the power cable, the energy variation of the capacitor $\Delta W_{c, \max }$ is $2.45 \times 10^{6} \mathrm{~J}$, and the energy variation of the SI-FCL A $\Delta W_{L, \max }$ is $2.48 \times 10^{6} \mathrm{~J}$. It can be seen from (10) that the SI-FCL has current-limiting

Table 3 Parameters of SI-FCL A

\begin{tabular}{ll}
\hline Parameter & Value \\
\hline Length of the core $(l)$ & $4 \mathrm{~m}$ \\
Cross-section areas $(S)$ & $0.6 \mathrm{~m}^{2}$ \\
Winding number of coil 1 $\left(N_{1}\right)$ & 6000 \\
Winding number of coil 2 $\left(N_{2}\right)$ & 900 \\
Current of the excitation coil $\left(i_{1}\right)$ & $150 \mathrm{~A}$ \\
Magnetic flux density of point $K\left(B_{k}\right)$ & $2.3 \mathrm{~T}$ \\
\hline
\end{tabular}


ability before the capacitor voltage drops to $U_{\mathrm{dc} \text {, min }}$. The SI-FCL A meets the condition of case 1 . Therefore, the magnetic flux density $B_{\min }$ is about $2.238 \mathrm{~T}$, the maximum fault current $i_{2, \text { max,I }}$ is $1.017 \mathrm{kA}$, and $T_{\text {min,I }}$ is about 40.2 ms. The above theoretical calculations were made for the most serious fault conditions (resistance of the DC side is 0 ). When a DC side fault occurs at other locations, the line resistance is greater than 0 , so the current-limiting effect of the SI-FCL can be better.

When a two-pole short-circuit fault occurs at $5 \mathrm{~km}$ away from the rectifier at $0.1 \mathrm{~s}$, the capacitor voltage and fault current of the VSC-HVDC system with SI-FCL A are shown in Figs. 9 and 10. It can be seen that the time $T$ for capacitor voltage to fall from $U_{\mathrm{dc} 1}$ to $U_{\mathrm{dc}, \min }$ is extended to $40.8 \mathrm{~ms}$ by the SI-FCL A. In addition, the maximum fault current is about $1.04 \mathrm{kA}$. The results obtained by the simulation and theoretical analysis of SI-FCL A are basically the same.

The parameters of SI-FCL B are listed in Table 4.

Assuming that the two-pole short-circuit fault occurs at the junction of the DC rail and the power cable in the VSCHVDC system with SI-FCL B. The energy variation of the capacitor $\Delta W_{c, \max }$ is $2.45 \times 10^{6} \mathrm{~J}$, and the energy variation of the SI-FCL B is $1.49 \times 10^{6} \mathrm{~J}$. It can be seen from (10) that the SI-FCL will lose current-limiting ability before the capacitor voltage drops to $U_{\mathrm{dc}, \min }$. The SI-FCL B meets the condition of case 2 , and the current surge occurs before the capacitor voltage drops to $U_{\mathrm{dc}, \min }$. The magnetic flux density $B_{k}$ substituted into (11) and (14), the maximum fault current before the current surges is about $1.389 \mathrm{kA}$, and the voltage $U_{\mathrm{dc}, \mathrm{s}}$ is $57.271 \mathrm{kV}$. The shortest time $T_{\mathrm{min}, \mathrm{s}}$ for the capacitor voltage to fall from $U_{\mathrm{dc} 1}$ to $U_{\mathrm{dc}, \mathrm{s}}$ is 18.3 ms. Assuming that the fault current remains $1.389 \mathrm{kA}$, the shortest time $T_{\min , \mathrm{s}}^{\prime}$ for capacitor voltage to fall from $U_{\mathrm{dc} 1}$ to $U_{\mathrm{dc}, \min }$ is $29.5 \mathrm{~ms}$. Therefore, the time for the capacitor voltage to drop to $U_{\mathrm{dc} \text {, min }}$ is longer than $18.3 \mathrm{~ms}$ and less than $29.5 \mathrm{~ms}$.

When a two-pole short-circuit fault occurs $5 \mathrm{~km}$ away from the rectifier at $0.1 \mathrm{~s}$, the capacitor voltage and fault

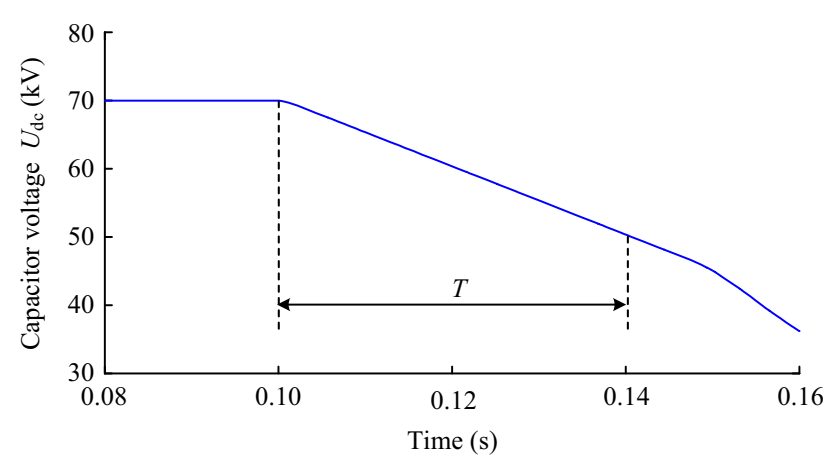

Fig. 9 Capacitor voltage of VSC-HVDC system with SI-FCL A

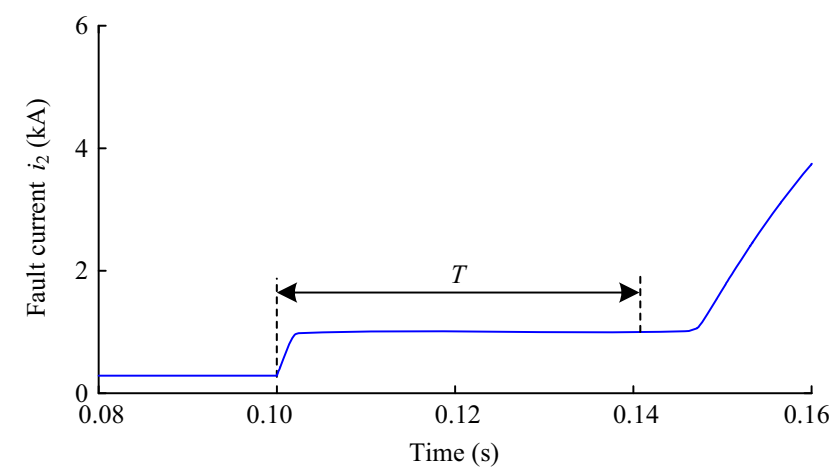

Fig. 10 DC side fault current of VSC-HVDC system with SI-FCL A

Table 4 Parameters of SI-FCL B

\begin{tabular}{ll}
\hline Parameter & Value \\
\hline Length of the core $(l)$ & $4 \mathrm{~m}$ \\
Cross-section areas $(S)$ & $0.6 \mathrm{~m}^{2}$ \\
Winding number of coil 1 $\left(N_{1}\right)$ & 4500 \\
Winding number of coil 2 $\left(N_{2}\right)$ & 400 \\
Current of the excitation coil $\left(i_{1}\right)$ & $120 \mathrm{~A}$ \\
Magnetic flux density of point $K\left(B_{k}\right)$ & $2.3 \mathrm{~T}$ \\
\hline
\end{tabular}

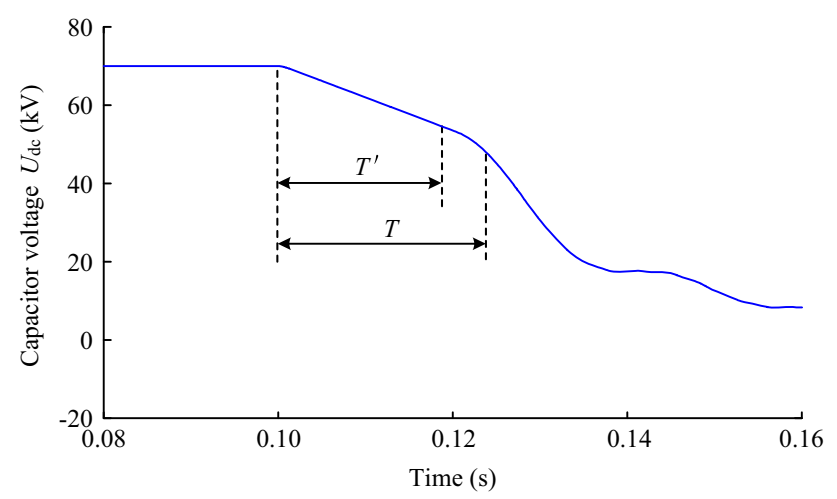

Fig. 11 Capacitor voltage of VSC-HVDC system with SI-FCL B

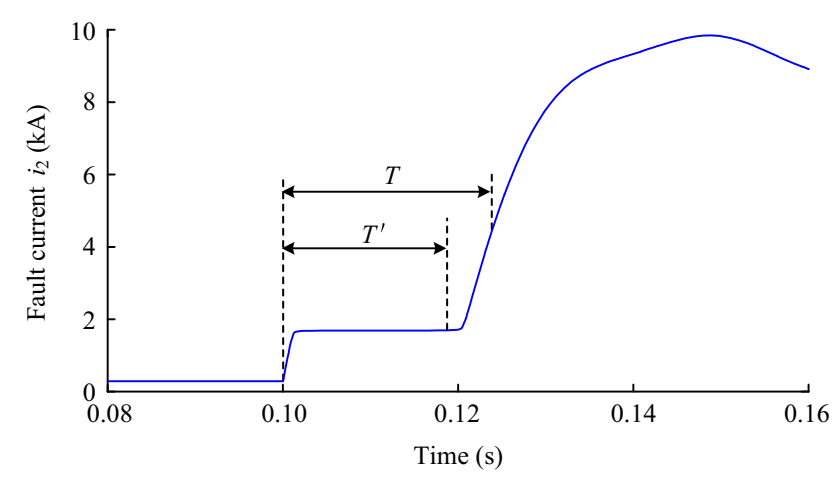

Fig. 12 DC side fault current of VSC-HVDC system with SI-FCL B 
current of the VSC-HVDC system with SI-FCL B are shown in Figs. 11 and 12 . It can be seen that the time $T$ for capacitor voltage to fall from $U_{\mathrm{dc} 1}$ to $U_{\mathrm{dc} \text {, min }}$ is $23.8 \mathrm{~ms}$, and the maximum fault current is approximately $1.409 \mathrm{kA}$ before the current surges. When the fault current surges, the capacitor voltage is $57.653 \mathrm{kV}$, and the time $T$ for capacitor voltage to fall from $U_{\mathrm{dc} 1}$ to $U_{\mathrm{dc}, \mathrm{s}}$ is $18.7 \mathrm{~ms}$. The results obtained by the simulation and theoretical analysis of SI-FCL B are basically the same.

The simulation of the VSC-HVDC system with different SI-FCLs shows that the method analyzing the performance of the SI-FCL based on the electromagnetic energy conversion mechanism is correct. The method gives the criterion for determining whether the SI-FCL has currentlimiting ability constantly before the capacitor voltage drops to $U_{\mathrm{dc} \text {,min. }}$ In addition, the maximum fault current and the time for capacitor voltage to fall from $U_{\mathrm{dc} 1}$ to $U_{\mathrm{dc} \text {,min }}$ can also be calculated accurately by this method.

\section{Conclusion}

This paper has examined the performance of the SI-FCL in a VSC-HVDC system. From the perspective of electromagnetic energy conversion, the relationship between the electromagnetic energy and the magnetic flux density of the SI-FCL has been analyzed. Based on the study of the electromagnetic energy conversion process after the DC side fault, the relationship between the fault current and the magnetic flux density of the SI-FCL and that between the time for the capacitor voltage dropping to $U_{\mathrm{dc}, \min }$ and the DC side fault current have been given.

The method identifying the current-limiting characteristic of the SI-FCL in the VSC-HVDC system is based on the analysis of the energy conversion of the DC side fault. On this basis, the time for capacitor voltage to drop to $U_{\mathrm{dc} \text {, min }}$ and the maximum fault current in the case of a constantly current-limiting-capable SI-FCL can be obtained if the parameters of the SI-FCL and the VSCHVDC system are given. This method avoids the complex differential calculation of solving the DC side fault transient process in the time domain, and its accuracy has been demonstrated through a case study and simulation.

Acknowledgements This research work was supported in part by the National Key R\&D Program of China (No. 2018YFB0904600), and in part by the National Nature Science Foundation of China (NSFC) under Grant 51677125 .

Open Access This article is distributed under the terms of the Creative Commons Attribution 4.0 International License (http:// creativecommons.org/licenses/by/4.0/), which permits unrestricted use, distribution, and reproduction in any medium, provided you give appropriate credit to the original author(s) and the source, provide a link to the Creative Commons license, and indicate if changes were made.

\section{References}

[1] Zeng D, Yao J, Yang S et al (2011) Economy comparison of VSC-HVDC with different voltage levels. Autom Electr Power Syst 35(20):98-102

[2] Elserougi AA, Abdel-Khalik AS, Massoud AM et al (2014) A new protection scheme for HVDC converters against DC-side faults with current suppression capability. IEEE Trans Power Deliv 29(4):1569-1577

[3] Yang J, Fletcher JE, O'Reilly J (2012) Short-circuit and ground fault analyses and location in VSC-based DC network cables. IEEE Trans Ind Electron 59(10):3827-3837

[4] Liu J, Tai NL, Fang CJ et al (2016) Fault current limitation and analysis of current limiting characteristic for multi-terminal VSC-HVDC DC lines. Proc CSEE 36(19):5122-5133

[5] Lee JG, Khan UA, Hwang JS et al (2014) Assessment on the influence of resistive superconducting fault current limiter in VSC-HVDC system. Phys C Supercond 504(1):163-166

[6] Manohar P, Ahmed W (2012) Superconducting fault current limiter to mitigate the effect of DC line fault in VSC-HVDC system. In: Proceedings of the 2012 IEEE international conference on power, signals, controls and computation, Thrissur, India, 3-6 January 2012, 6 pp

[7] Liu X, Sheng J, Hong Z et al (2015) Research on application of superconducting fault current limiter in VSC-MTDC power system. Power Syst Technol 39(4):983-988

[8] Kumar MA, Babu SS, Srikanth NV et al (2015) Performance of superconducting fault current limiter in offshore wind farm connected VSC based MTDC transmission system. In: Proceedings of the IEEE power and energy conference, Champaign, USA, 20-21 February 2015, 6 pp

[9] Hayakawa N, Maeno Y, Kojima H (2018) Fault current limitation coordination in electric power grid with superconducting fault current limiters. IEEE Trans Appl Supercond. https://doi. org/10.1109/TASC.2018.2818279

[10] Chen Y, Liu X, Sheng J et al (2014) Design and application of a superconducting fault current limiter in DC systems. IEEE Trans Appl Supercond 24(3):1-5

[11] Li B, He J (2015) DC fault analysis and current limiting technique for VSC based DC distribution system. Proc Chin Soc Electr Eng 35(12):3026-3036

[12] Wang C, Li B, He J et al (2017) Design and application of the SFCL in the modular multilevel converter based DC system. IEEE Trans Appl Supercond 27(4):1-4

[13] Descloux J, Raison B, Curis JB (2014) Protection algorithm based on differential voltage measurement for MTDC grids. In: Proceedings of the IET international conference on developments in power system protection, Copenhagen, Denmark, 31 March-3 April 2014, 5 pp

[14] Kontos E, Rodrigues S, Teixeira PR et al (2014) Optimization of limiting reactors design for DC fault protection of multi-terminal HVDC networks. In: Proceedings of energy conversion congress and exposition, Pittsburgh, USA, 14-18 September 2014, 8 pp

[15] Deng F, Chen Z (2013) Design of protective inductors for HVDC transmission line within DC grid offshore wind farms. IEEE Trans Power Deliv 28(28):75-83

[16] Li B, Wang C, Wei $Z$ et al (2018) Technical requirements of the DC superconducting fault current limiter. IEEE Trans Appl Supercond. https://doi.org/10.1109/TASC.2018.2811961 
[17] Li B, Jing F, Jia F et al (2016) Research on saturated iron-core superconductive fault current limiters applied in VSC-HVDC systems. IEEE Trans Appl Supercond 26(7):1-4

[18] Zhang C, Tang Y, Xu Y et al (2017) Analysis of magnetic circuit and leakage magnetic field of a saturated iron-core superconducting fault current limiter. IEEE Trans Appl Supercond 27(4):1-5

[19] Li B, Jing F, Li B et al (2018) Study of the application of active saturated iron-core superconductive fault current limiters in the VSC-HVDC system. IEEE Trans Appl Supercond. https://doi. org/10.1109/TASC.2018.2824840

[20] Aracil JC, Lopez-Roldan J, Coetzee JC et al (2012) Analysis of electromagnetic forces in high voltage superconducting fault current limiters with saturated core. Int J Electr Power Energy Syst 43(1):1087-1093

[21] Jia Y, Shi Z, Zhu H et al (2015) Cognition on the currentlimiting effect of saturated-core superconducting fault current limiter. IEEE Trans Magn 51(11):1-4

[22] Li B, Guo F, Wang J et al (2015) Electromagnetic transient analysis of the saturated iron-core superconductor fault current limiter. IEEE Trans Appl Supercond 25(3):1-5

[23] Feng CZ, Ma XK (2001) An introduction to engineering electromagnetic fields. Higher Education Press, Beijing

Botong LI received the Ph.D. degree in electrical engineering and automation from Tianjin University, Tianjin, China in 2010. He is an associate professor in the School of Electrical and Information Engineering, Tianjin University, Tianjin, China. His research interests include power system protection and control of VSC-HVDC transmission system.

Hanqing CUI is currently pursuing the M.S. degree in electrical engineering and automation at Tianjin University, Tianjin, China. His research interests include protection of VSC-HVDC.

Fangjie JING is currently pursuing the M.S. degree in electrical engineering and automation at Tianjin University, Tianjin, China. His research interests include protection of VSC-HVDC.

Bin LI received the Ph.D. degree in electrical engineering and automation from Tianjin University, Tianjin, China in 2005. He is currently a professor in the School of Electrical and Information Engineering, Tianjin University, Tianjin, China. His research interests include power system protection and control.

Yichao LIU is currently pursuing the M.S. degree in electrical engineering and automation at Tianjin University, Tianjin, China. His research interests include protection of VSC-HVDC. 